\title{
Isolated Central Nervous System Blast Crisis in Chronic Myeloid Leukemia - Report of Two Cases
}

\begin{abstract}
Extramedullary blast crisis (BC) presenting as the central nervous system (CNS) involvement in chronic myeloid leukemia (CML) is rare and usually accompanies systemic involvement. Imatinib has shown efficacy in treating patients with CML in accelerated or blastic phases. However, CNS penetration of Imatinib is poor due to the P-glycoprotein mediated efflux mechanisms. Therefore, patients on long-term Imatinib therapy with hematological and cytogenetic remission may rarely present with CNS BC. Isolated CNS BC is uncommon and the literature is limited to only a few case reports. Here, we present two cases of isolated CNS BC in patients with BCR ABL positive CML on treatment.
\end{abstract}

Keywords: Chronic myeloid leukemia, central nervous system blast crisis, extramedullary blast crisis

\section{Introduction}

Tyrosine kinase inhibitors (TKIs) have dramatically improved the prognosis and survival of patients with chronic myeloid leukemia (CML) and have decreased the chances of progression to blast crisis (BC) ${ }^{[1]}$ Extramedullary $\mathrm{BC}$ (EBC) can occur as an isolated event in $5 \%-10 \%$ of patients; however, the central nervous system (CNS) as a site of $\mathrm{EBC}$ is extremely rare. ${ }^{[2,3]}$ With Imatinib, the majority of patients are expected to achieve a complete cytogenetic response. ${ }^{[2]}$ However, the CNS penetration of Imatinib is poor due to P-glycoprotein mediated efflux mechanism. ${ }^{[4]}$ Here, we describe two cases of isolated CNS BC in patients with BCR-ABL fusion (BCR - Breakpoint Cluster Region; ABL - Abelson gene) positive CML on treatment.

\section{Case Reports}

Case presentation of isolated central nervous system relapse in patient on Imatinib (Case 1)

A 68-year-old male was diagnosed with CML with p210 b3a2 BCR ABL transcript positivity in May 2017. Bone marrow aspirate (BMA) was suggestive of CML

\footnotetext{
This is an open access journal, and articles are distributed under the terms of the Creative Commons Attribution-NonCommercialShareAlike 4.0 License, which allows others to remix, tweak, and build upon the work non-commercially, as long as appropriate credit is given and the new creations are licensed under the identical terms.
}

For reprints contact: WKHLRPMedknow reprints@wolterskluwer.com in chronic phase (CP). The patient was started on tablet Imatinib $400 \mathrm{mg}$ once daily (OD). The quantitative BCR ABL levels at $3^{\text {rd }}, 6^{\text {th }}$ and $12^{\text {th }}$ months were $18 \%$, $6.8 \%$ and $16.8 \%$ international scale (IS), respectively. The dose was reduced to $300 \mathrm{mg}$ after completion of 1 year due to multiple mucosal ulcers probably lichenoid eruptions. ${ }^{[5,6]}$

On completion of 20 months of treatment, he presented with headache, vomiting and altered sensorium. Computed tomography (CT) of the brain was normal. The BCR $\mathrm{ABL}$ quantitative polymerase chain reaction (PCR) was $23.9 \%$ IS. The patient also had T315I mutation in kinase domain mutation analysis. Cerebrospinal fluid (CSF) sample morphology showed pleocytosis with myeloid hyperplasia, left shift and $35 \%$ blasts [Figure 1a]. Flow cytometry (FCM) performed in the CSF sample showed $30 \%$ blasts with an expression of CD13 dim to moderate, CD33 moderate, CD15 moderate, CD117 dim to moderate, bright myeloperoxidase (MPO) suggestive of CML myeloid BC [Figure 2a]. BCR ABL fluorescence in situ hybridization performed on the CSF sample showed $83 \%$ of nuclei with BCR ABL positivity [Figure 3]. BMA and biopsy performed were suggestive of CML CP. These features suggested an isolated CNS myeloid BC.

\footnotetext{
How to cite this article: Arumugam JR Karthik Bommannan BK, Kalaiyarasi JP, Sundersingh $\mathrm{S}$. Isolated central nervous system blast crisis in chronic myeloid leukemia - Report of two cases. Indian J Med Paediatr Oncol 2020;41:781-3.
}

\author{
Jhansi Rani \\ Arumugam ${ }^{1}$, \\ Karthik \\ Bommannan B.K. ${ }^{1}$, \\ Jayachandran \\ Perumal \\ Kalaiyarasi ${ }^{2}$, \\ Shirley \\ Sundersingh ${ }^{1}$
}

${ }^{\prime}$ Department of Oncopathology, Cancer Institute (WIA), Chennai, Tamil Nadu, India,

${ }^{2}$ Department of Medical Oncology, Cancer Institute (WIA), Chennai,

Tamil Nadu, India

Submitted: 14-May-2020 Accepted in Revised Form: 17-May-2020

Published: 29-Oct-2020

Address for correspondence: Dr. Jhansi Rani Arumugam, Department of Oncopathology, Cancer Institute (WIA), Adyar, Chennai - 600036 ,

Tamil Nadu, India.

E-mail:drjhansi5@gmail.com

Access this article online

Website: www.ijmpo.org

DOI: $10.4103 /$ /jmpo.ijmpo 233_20 Quick Response Code:

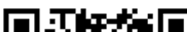
Ifory 4.275 trescon 口ingt: 
The patient was given five biweekly triple intrathecal (TIT) injections (Methotrexate $12 \mathrm{mg}$, Hydrocortisone $30 \mathrm{mg}$ and Cytarabine $50 \mathrm{mg}$ ) with Imatinib $400 \mathrm{mg}$ OD. Further CSF analysis showed a decreasing pattern of counts and blasts and improvement of symptoms. Subsequently, patient deteriorated and expired within few days.

Case presentation of isolated central nervous system relapse in patient on Nilotinib (Case 2)

A 22-year-old male diagnosed as CML-CP with $\mathrm{t}(9 ; 22)$ (q34;q11) by cytogenetics. He was started on tablet Imatinib $400 \mathrm{mg}$. He attained complete hematologic remission (CHR) in 4 months and continued Imatinib 400 mg OD till 2015. Then, he defaulted treatment for $3 \frac{1}{2}$ years in between and came back with a loss of CHR and BCR ABL level was $17.5 \%$ IS. Imatinib was restarted at a dose of $600 \mathrm{mg}$ OD.

Subsequently, after few months he presented with bleeding tendencies, during which BMA showed $57 \%$ blasts. FCM performed was consistent with myeloid $\mathrm{BC}$ with blast population expressing CD13, CD16, CD11b, CD34 and HLA-DR. The patient was started on tablet Nilotinib 400 $\mathrm{mg} \mathrm{BD}$, and then, the dose was reduced to $200 \mathrm{mg} \mathrm{BD}$ in view of thrombocytopenia. Clinically, patient improved and achieved major molecular response (BCR- ABL of $0.02 \%$ IS) after 18 months of Nilotinib.

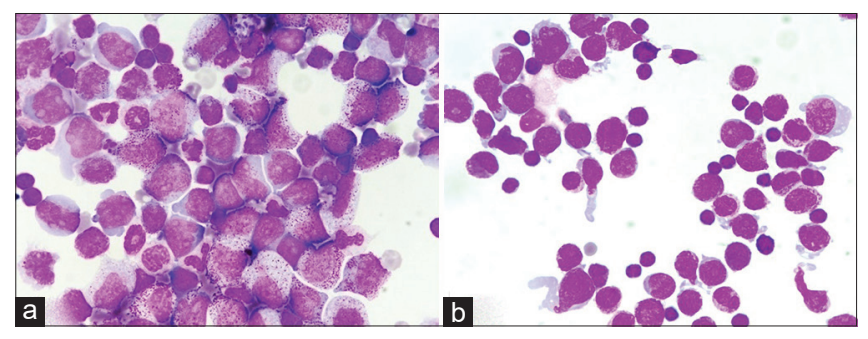

Figure 1: (a and b) Cerebrospinal fluid showing increased blasts of case 1 and 2, respectively, (Leishman $\times 1000$ )
Subsequently, the patient came with complaints of headache and blurring of vision within 2 months. The hemogram was normal. BMA was diluted with $2 \%$ blasts. The BCR - ABL Quantitative PCR was $0.005 \%$ IS. CT scan of the brain showed ill-defined hypodense lesion in the frontal lobe. Magnetic resonance imaging of the brain showed hyperintense signal in the left frontal lobe and suggested CSF analysis. CSF analysis performed showed hypercellular smears showing myeloid hyperplasia with left shift, basophilia, and $45 \%$ blasts [Figure 1b]. FCM performed in the CSF sample showed $40 \%$ blasts with moderate expression of CD34, CD38, CD58, HLA-DR, CD123, CD33, and $\mathrm{CD} 7$, negative to dim expression of $\mathrm{CD} 13$ and negative for CD10, CD19, CD20, cytoplasmic MPO, CD3 and CD79 [Figure 2b]. Features were consistent with myeloid $\mathrm{BC}$ in CNS in a known case of CML in molecular remission. The patient was started on biweekly TIT injections along with Dasatinib tablets at the dose of $100 \mathrm{mg}$ once daily. Despite the clearance of blasts in the subsequent CSF analyses, the patient continued to have have progressive loss of vision and was then lost to follow up.

\section{Discussion}

$\mathrm{EBC}$ with CNS involvement is rare and usually accompanies systemic involvement. ${ }^{[7]}$ Theoretically, circulating leukemic cells enter the brain or meninges by five different routes, of which hematogenous spread is the most important mode of spread ${ }^{[8]}$ Imatinib can induce a hematological response in over $95 \%$ of patients and major cytogenetic responses in $85 \%$ of the patients. It has also shown activity in CML in accelerated or blastic phases. ${ }^{[7]}$ However, CNS penetration of Imatinib is poor (CSF concentration $<1 \%$ that of the plasma) due to the P-glycoprotein mediated efflux mechanisms. Patients on long-term Imatinib therapy with cytogenetic remission may rarely present with CNS BC. Such a diagnosis of isolated CNS BC is rare, and the literature is limited to few case reports. ${ }^{[3,9]}$ Various efflux

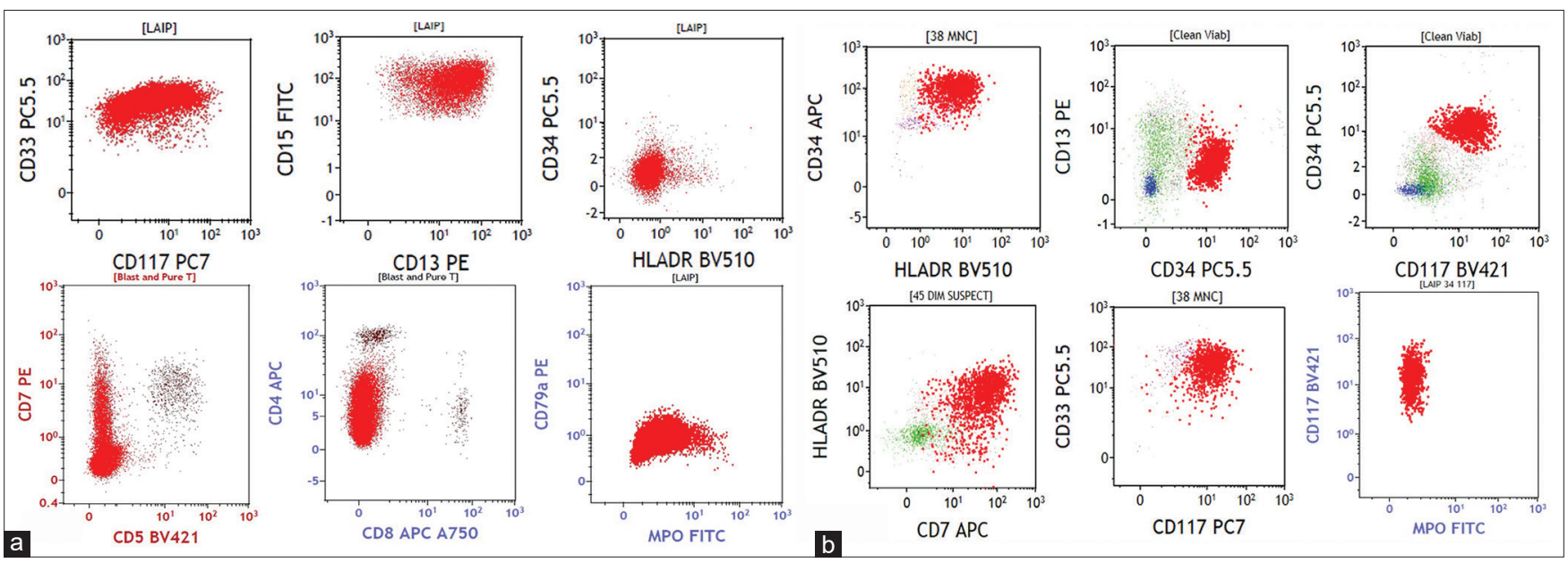

Figure 2: (a) Flow cytometry of case 1 showing blasts (red population) expressing CD33, CD15, CD13, CD7 and myeloperoxidase and negative for CD34, HLA-DR, CD5, CD4, CD8 and CD79. (b) Flow cytometry of case 2 showing blasts (red population) expressing CD34, HLA-DR, CD13, CD117, CD7, CD33 and negative for myeloperoxidase 


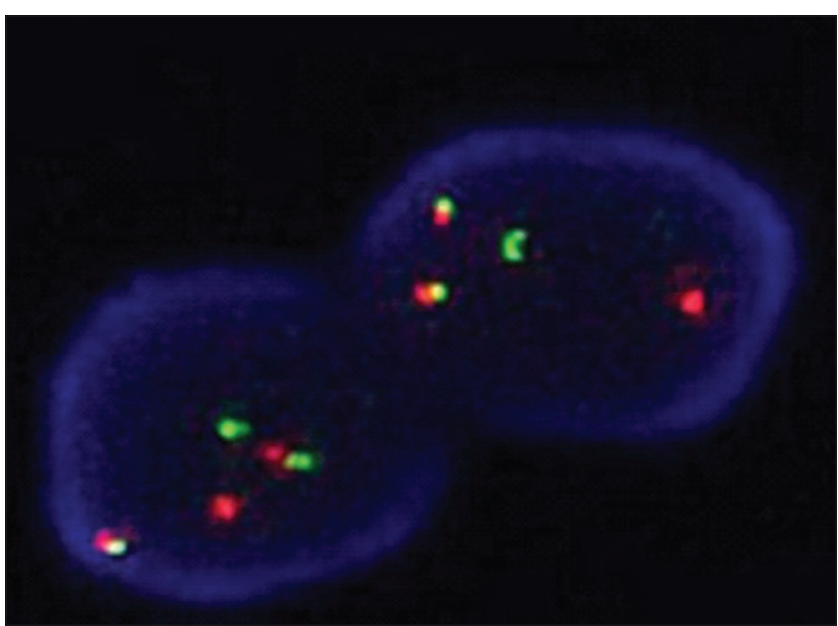

Figure 3: Case 1 positive for BCR ABL dual color dual fusion fluorescence in situ hybridization with two fusions, one green and one orange signal

transporters are expressed in the blood brain barrier and blood-CSF barrier that eliminate compounds from the brain or CSF to the blood. P-glycoprotein is expressed on the apical side of the brain capillary endothelium and can transport substrates from the basolateral side (brain) to the apical side (blood) of the blood brain barrier and thus limit the brain distribution and decrease the specific brain tissue bioavailability of many therapeutic agents. ${ }^{[4]}$

Treatment of such cases includes intrathecal therapy along with a second-line TKIs such as Dasatinib which is known to have better CNS penetration. Dasatinib has an effective therapeutic potential in managing intracranial leukemic disease in patients who experience CNS relapse while on Imatinib therapy. ${ }^{[10,11]}$ Allogeneic stem cell transplantation (SCT) was found to improve the survival of patients after successful CNS treatment compared to patients who were not transplanted. ${ }^{[9]}$

In our cases, despite Imatinib and Nilotinib, the disease had progressed in CNS, which highlights the pharmacokinetics of these drugs and the importance of outcomes to be considered in patients on long-term therapy.

\section{Conclusion}

Isolated $\mathrm{CNS} \mathrm{BC}$ in $\mathrm{CML}$ is a rare phenomenon. CNS penetration of Imatinib is poor due to the P-glycoprotein mediated efflux mechanisms. Hence, it is mandatory to identify such cases and treat them with appropriate TKIs, intrathecal therapy followed by SCT.

Financial support and sponsorship

Nil.

\section{Conflicts of interest}

There are no conflicts of interest.

\section{References}

1. Ishii K, Nakaya A, Fujita S, Satake A, Azuma Y, Tsubokura Y, et al. Myodesopsia is a symptom of central nervous system blast crisis in chronic myeloid leukemia. Leuk Res Rep 2019;11:8-10.

2. Rajappa S, Uppin SG, Raghunadharao D, Rao IS, Surath A. Isolated central nervous system blast crisis in chronic myeloid leukemia. Hematol Oncol 2004;22:179-81.

3. Lindhorst SM, Lopez RD, Sanders RD. An unusual presentation of chronic myelogenous leukemia: A review of isolated central nervous system relapse. J Natl Compr Canc Netw 2013;11:745-9.

4. Dai H, Marbach P, Lemaire M, Hayes M, Elmquist WF. Distribution of STI-571 to the brain is limited by P-glycoproteinmediated efflux. J Pharmacol Exp Ther 2003;304:1085-92.

5. Ena P, Chiarolini F, Siddi GM, Cossu A. Oral lichenoid eruption secondary to imatinib (Glivec). J Dermatolog Treat 2004;15:253-5.

6. Lim DS, Muir J. Oral lichenoid reaction to imatinib (STI 571, Gleevec). Dermatology 2002;205:169-71.

7. Altintas A, Cil T, Kilinc I, Kaplan MA, Ayyildiz O. Central nervous system blastic crisis in chronic myeloid leukemia on imatinibmesylate therapy: A case report. J Neurooncol 2007;84:103-5.

8. Thomas LB. Pathology of leukemia in the brain and meninges: Postmortem studies of patients with acute leukemia and of mice given inoculations of L1210 leukemia. Cancer Res 1965;25:1555-71.

9. Jain A, Gupta N. Isolated CNS blast crises in chronic myeloid leukaemia presenting as hypertrophic pachymeningitis and bilateral optic neuritis: A case report. J Clin Diagn Res 2016;10:OE01-5.

10. Morillo LA, Zapata-Mesina F, Castillo MR. A case of isolated CNS relapse in a CML patient on chronic phase. IBRR 2015;4:1-8.

11. Porkka K, Koskenvesa P, Lundán T, Rimpiläinen J, Mustjoki S, Smykla R, et al. Dasatinib crosses the blood-brain barrier and is an efficient therapy for central nervous system Philadelphia chromosome-positive leukemia. Blood 2008;112:1005-12. 